\title{
Long-Term Asthma Trend Monitoring in New York City: A Mixed Model Approach
}

\author{
Stephen E. Schachterle*, Robert W. Mathes, Marc Paladini and Don Weiss \\ New York City Department of Health and Mental Hygiene, Queens, NY, USA
}

\section{Objective}

Show the benefits of using a generalized linear mixed model (GLMM) to examine long-term trends in asthma syndrome data.

\section{Introduction}

Over the last decade, the application of syndromic surveillance systems has expanded beyond early event detection to include longterm disease trend monitoring. However, statistical methods employed for analyzing syndromic data tend to focus on early event detection. Generalized linear mixed models (GLMMs) may be a useful statistical framework for examining long-term disease trends because, unlike other models, GLMMs account for clustering common in syndromic data, and GLMMs can assess disease rates at multiple spatial and temporal levels (1). We show the benefits of the GLMM by using a GLMM to estimate asthma syndrome rates in New York City from 2007 to 2012, and to compare high and low asthma rates in Harlem and the Upper East Side (UES) of Manhattan.

\section{Methods}

Asthma related emergency department (ED) visits, and patient age and ZIP code were obtained from data reported daily to the NYC Department of Health and Mental Hygiene. Demographic data were obtained from 2010 US Census. ZIP codes that represented high and low asthma rates in Harlem and the UES of Manhattan were chosen for closer inspection. The ratio of weekly asthma syndrome visits to total ED visits was modeled with a Poisson GLMM with week and ZIP code random intercepts (2). Age and ethnicity were adjusted for because of their association with asthma rates (3).

\section{Results}

The GLMM showed citywide asthma rates remained stable from 2007 to 2012, but seasonal differences and significant inter-ZIP code variation were present. The Harlem ZIP code asthma rate that was estimated with the GLMM was significantly higher $(5.83 \%, 95 \% \mathrm{CI}$ : $3.65 \%, 9.49 \%)$ than the asthma rate in UES ZIP code $(0.78 \%, 95 \% \mathrm{CI}$ : $0.50 \%, 1.21 \%$ ). A linear time component to the GLMM showed no appreciable change over time despite the seasonal fluctuations in asthma rate. GLMM based asthma rates are shown over time (Figure 1).

\section{Conclusions}

GLMMs have several strengths as statistical frameworks for monitoring trends including:

1. Disease rates can be estimated at multiple spatial and temporal levels,

2. Standard error adjustment for clustering in syndromic data allows for accurate, statistical assessment of changes over time and differences between subgroups,

3. "Strength borrowed" (4) from the aggregated data informs small subgroups and smooths trends,

4. Integration of covariate data reduces bias in estimated rates.

GLMMs have previously been suggested for early event detection with syndromic surveillance data (5), but the versatility of GLMM makes them useful for monitoring long-term disease trends as well. In comparison to GLMMs, standard errors from single level GLMs do not account for clustering and can lead to inaccurate statistical hypothesis testing. Bayesian hierarchical models (6), share many of the strengths of GLMMS, but are more complicated to fit. In the future, GLMMs could provide a framework for grouping similar ZIP codes based on their model estimates (e.g. seasonal trends and influence on overall trend), and analyzing long-term disease trends with syndromic data.

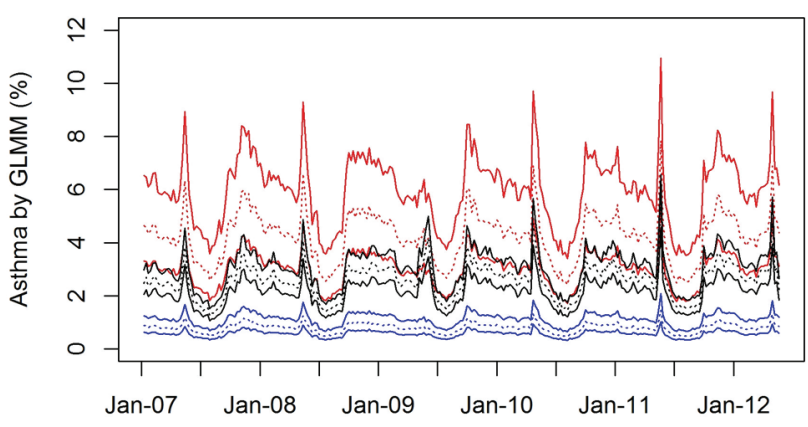

Figure 1: Harlem ZIP code (red), the Upper East Side ZIP code (blue), and citywide (black) estimates are shown as dotted lines surrounded by $30 \%$ credibility bands in solid lines.

\section{Keywords}

Asthma; Long term trends; Generalized Mixed Models

\section{Acknowledgments}

This work was carried out in conjunction with a grant from the Alfred P. Sloan Foundation (\#2010-12-14). We thank the members of the New York City Department of Health and Mental Hygiene Syndromic Surveillance Unit.

\section{References}

1. Diggle, P.J., Liang K.Y., \& Zeger, S. L. (1994). Analysis of Longitudinal Data. Oxford: Oxford University Press.

2. Gelman, A., Hill, J., (2007). Data Analysis Using Regression and Multilevel/Hierarchical Models. New York: Cambridge University Press.

3. Claudio, L. et al. (1999) Socioeconomic Factors and Asthma Hospitalization Rates in New York City. Journal of Asthma. 36(4): pp. 343350 .

4. Jones, K., Johnston R. J., and Pattie C. J. (1992). People, Places and Regions: Exploring the Use of Multi-Level Modelling in the Analysis of Electoral Data. British Journal of Political Science, 22, pp 343-380.

5. Kleinman, K., Lazarus, R., Platt, R., (2004). A Generalized Linear Mixed Models Approach for Detecting Incident Clusters of Disease in Small Areas, with an Application to Biological Terrorism. AJE, 159, pp 217-224.

6. Chan, T.C., et al. Probabilistic Daily ILI Syndromic Surveillance with a Spatio-Temporal Bayesian Hierarchical Model. PLoS ONE. 5(7): e11626.

\footnotetext{
*Stephen E. Schachterle

E-mail: steve.schachterle@gmail.com
} 